\title{
Confinement of 'Improved H-Modes' in the All-Tungsten ASDEX Upgrade with Nitrogen Seeding
}

J. Schweinzer 1), A.C.C. Sips 2), G. Tardini 1), P.A. Schneider 1), R. Fischer 1), J.C. Fuchs 1), O. Gruber 1), J. Hobirk 1), A. Kallenbach 1), R. M. McDermott 1), R. Neu 1), T. Pütterich 1), S. K. Rathgeber 1), J. Stober 1), J. Vicente 3) and the ASDEX Upgrade Team

1) Max-Planck-Institut für Plasmaphysik, D-85748 Garching, Germany, Euratom Association

2) EFDA-JET, Culham Science Centre, OX14 3DB, Abingdon, UK

3) Associacao EURATOM/IST, Instituto de Plasmas e Fosao Nuclear - Laboratorio Associado, IST, Lisbon, Portugal

E-mail contact of main author: josef.schweinzer@ipp.mpg.de

\begin{abstract}
In ASDEX Upgrade the compatibility of improved H-modes with an all-W wall has been demonstrated. Under boronized conditions light impurities and the radiated power fraction in the divertor were reduced, requiring $\mathrm{N}$ seeding to cool the divertor plasma. The impurity seeding does not only protect the divertor tiles but also considerably improves the performance of improved $\mathrm{H}$-mode discharges by up to $25 \%$. The energy confinement increases to $\mathrm{H}_{98}$-factors up to 1.3 and thereby exceeds the best values in the carbon-dominated AUG at the same density and collisionality. This improvement is due to higher edge temperatures rather than to peaking of the electron density profile. Higher temperatures are reached at the pedestal top leading, via profile stiffness, to an increase of the total plasma pressure. There is no change to $\mathrm{R} / \mathrm{L}_{\mathrm{Te}, \mathrm{i}}$ in the plasma core. The dilution at the plasma edge by nitrogen seems to play an important role since it allows higher ion temperatures at the same edge ion pressure as in the unseeded case. The dilution of the core plasma remains moderate.
\end{abstract}

PACS number: 52.55.Fa

\section{Introduction}

'Improved H-mode' discharges (IPHM) in ASDEX Upgrade (AUG) are characterized by enhanced confinement factors $\mathrm{H}_{98}>1$, improved MHD-stability, a total beta $\beta_{\mathrm{N}}=2-3.5$ and a q-profile with a broad region in the plasma core of $\sim$ zero shear and a $q(0) \approx 1$ [1]. This scenario, which is also often called a Hybrid scenario, opens the way in ITER to either longer 
plasma pulse duration at reduced plasma current and $\mathrm{Q}=10$ or to improved performance with $\mathrm{Q}>10$ at full plasma current. Hybrid scenarios typically require moderate additional heating during the plasma current ramp-up to allow the formation of an adequate current profile (low central shear) in preparation for strong heating during the flat-top phase. This current ramp-up phase is thought to be crucial for improved H-mode operation. However, alternative current ramp-up scenarios were developed such as an explicit 'late heating' scheme with a long ohmic phase at low plasma density before adding auxiliary heating. Surprisingly this resulted in better energy confinement compared with the 'early heating' scheme, with the discharge evolving to have low magnetic shear in the core during the high beta phase by means of the edge bootstrap current [1].

One of the major goals of the AUG tungsten programme has been to demonstrate the compatibility of such high performance scenarios with an all-W first-wall and divertor. Beginning in 1999, the plasma facing components (PFCs) in AUG have been changed in a stepwise manner from carbon to tungsten coated tiles [2]. Since 2007 all PFCs are coated with tungsten. The paper is organized as follows: Sec. II compares the achieved performance of improved H-modes in the all-W AUG with results from previous campaigns and describes the operational boundary conditions to be respected with the all-metal wall. Sect. III deals with the observed confinement improvement in nitrogen seeded plasmas. Finally, Sect. 4 summaries the results and compares them with recent impurity seeding studies on other machines like JET and ALCATOR C-Mod.

\section{Comparison of IPHM in the all-W AUG with results from previous campaigns}

During the last decade, improved H-mode operation at AUG has been achieved for a wide range of deuterium plasmas using predominantly neutral beam injection (NBI) with heating powers up to $15 \mathrm{MW}$. However, in order to obtain stationary conditions in AUG with its Wcoated tiles, the $\mathrm{W}$ concentration in the plasma needs to be controlled. In an H-mode, the edge transport barrier provides a zone of good confinement, and the inward transport of tungsten is regulated by the ELMs. Thus, a higher ELM frequency is beneficial for reducing the tungsten concentration [3]. The ELM frequency increases with the amount of additional heating, the edge $\mathrm{q}_{95}$ and the applied gas puff level. However, too high levels of fuelling deteriorate the global energy confinement. Thus, optimization of the plasma performance requires a control of the $\mathrm{W}$ concentration, while maintaining good confinement. In the core of the plasma, the neo-classical inward flow of $\mathrm{W}$ can lead to accumulation of tungsten which is caused by density peaking at high confinement conditions. Therefore, plasma heating needs to be 
provided by NBI and RF heating to produce sufficient central heat flux to enhance the turbulent transport in the plasma core [4]. In particular, in the all-W AUG a careful combination of these tools (gas puff and localized central heating) is a prerequisite to achieve stable operation with tolerable core $\mathrm{W}$ concentration below $5 \times 10^{-5}$. The mandatory gas puffing in the all-W AUG results in an operation at higher densities / collisonalities compared to earlier campaigns with a carbon dominated wall or during the transition phase (2001-2006) with an incomplete $\mathrm{W}$ coverage (see fig. 1).

In all of the campaigns between 2001 and 2006, ion cyclotron resonance heating (ICRH) was applied at 30 or $36 \mathrm{MHz}$ using a hydrogen minority heating scheme with a maximum coupled power of 6MW. During AUG's transition phase from a carbon to a $\mathrm{W}$ machine ICRH was a very effective central heating method. Since 2007, the use of ICRF heating in the all-W AUG is hampered by ICRF-related impurity sources from W-coated plasma facing components close to the antennas [5]. Since the installation of W-coated antenna limiters, ICRF heating is accompanied by a strong $\mathrm{W}$ release, which obliterates the beneficial effect of central heating. Therefore, ICRH was not applied to IPHMs in 2008/9 and ECRH remains the only RF heating method available to supply the required central heating for the suppression of central W accumulation. One long pulse (10s) 1MW gyrotron was available during 2008/9. Four smaller units which provide $500 \mathrm{~kW} / 2 \mathrm{~s}$ each have been available as well. Central deposition with the $140 \mathrm{GHz}$ gyrotrons restricted the operation to magnetic fields $\mathrm{B}_{\mathrm{t}}$ between $2.45 \mathrm{~T}$ and $2.55 \mathrm{~T}$ and to central electron densities $\mathrm{n}_{\mathrm{e}}$ below $1.2 \cdot 10^{20} \mathrm{~m}^{-3}$.

The all-W AUG was operated in an initial phase without applying boronization for vessel conditioning in order to allow the characterisation of the new tungsten wall, in particular the hydrogen retention studies. Despite the removal of all macroscopic carbon sources, the carbon content in AUG was not strongly reduced. In April 2008 this initial period ended and AUG was boronized again to enlarge its operational space by reducing the concentration of light impurities such as carbon and oxygen $(\mathrm{C}: 0.1-1 \%, \mathrm{O}<0.1 \%)$. The radiated power decreased, especially in the divertor, and the thermal load on the $\mathrm{W}$-coated divertor tiles reached values of more than $10 \mathrm{MW} / \mathrm{m}^{2}$ [6], which is beyond the power handling capabilities of $\mathrm{W}$ coatings. As a consequence, discharges in the boronized AUG with heating power above 10MW could be conducted in a safe manner only with active cooling of the divertor plasma by enhancing the radiation with $\mathrm{N}$ seeding. The amount of puffed $\mathrm{N}_{2}$ is feedback controlled by requesting a low divertor temperature, which is estimated by measuring the thermo-electric currents in the divertor [7]. As a positive surprise it turned out that puffing nitrogen does not only protect the divertor tiles, but also enhances significantly the confinement of the discharge [4]. The 
performance improvement with nitrogen seeding is very reproducible, holds for all $\mathrm{D}_{2}$ fuelling rates under both freshly boronized as well as unboronized conditions, and enhances the energy confinement by up to $25 \%$.

Fig. 1 shows improved H-mode data from 2008/9 all-W campaigns in comparison with similar discharges of previous campaigns. The normalized H-mode confinement factor $\mathrm{H}_{98(\mathrm{y}, 2)}$ is shown versus line-averaged electron density $\mathrm{n}_{\mathrm{e}}(\mathrm{left})$ and collisionality $v_{e}^{*}$ (right) for 'early' (full symbols) and 'late' (crosses) heated improved H-mode discharges with plasma currents of $1 \mathrm{MA}$ at small/medium triangularity $(0.23<\delta<0.3)$ with a $\mathrm{q}_{95} \sim 4.6$. In fig. 1 , no distinction has been made between boronized and unboronized wall. Each data point represents an average over a phase around the highest $\mathrm{W}_{\mathrm{MHD}_{-} \max }\left(\mathrm{W}_{\mathrm{MHD}}>0.85 \cdot \mathrm{W}_{\mathrm{MHD}_{-} \max }\right)$ of a single discharge. Our criteria is that, such high performance phases have to last at least for three energy confinement times, $\tau_{\mathrm{E}}$. A volume averaged definition of $v_{e}^{*}[8]$ has been used in fig. 1 involving the thermal stored energy $\mathrm{W}_{\text {th }}$ rather than explicit temperature and density profile data. The presented discharges have a total additional heating power between $5-15 \mathrm{MW}$ and have at least $0.8 \mathrm{MW}$ of ECRH power. Discharges from the 2008/9 campaigns (all-W AUG) are with $\mathrm{D}_{2}$ fuelling $\left(1.0 \cdot 10^{21}-1.2 \cdot 10^{22} \mathrm{~s}^{-1}\right)$ while discharges from 2001 to 2006 have no gas fuelling except a single one $\left(7 \cdot 10^{21} \mathrm{~s}^{-1}, \mathrm{H}_{98 \mathrm{y} 2}=1.1, \mathrm{n}_{\mathrm{e}}=7 \cdot 10^{19} \mathrm{~m}^{-3}\right)$. The presented data covers a range in Greenwald fraction $\mathrm{f}_{\mathrm{GW}}$ from 0.35 to 0.75 (see left figure).

In fig. 1, the results of the 2008/9 all-W campaigns are at higher plasmas density compared to previous campaigns. The envelope of $\mathrm{H}$-factors $\mathrm{H}_{98(\mathrm{y}, 2)}$ for early heated IPHMs follows approximately a curve $\left(\mathrm{H}_{98(\mathrm{y}, 2)} \propto \mathrm{n}_{\mathrm{e}}^{-0.41}\right)$ representing the inverse density behaviour of the $\mathrm{H}$ mode scaling $\left(\tau_{98(y, 2)} \propto \mathrm{n}_{\mathrm{e}}^{0.41}\right)$. Late heated IPHMs show better confinement than the early heated ones.

Using collisionality $v_{e}^{*}$ (right plot of fig. 1) instead of electron $n_{\mathrm{e}}$ (left plot of fig. 1) seems to sort the confinement data points better and bridges between carbon dominated campaigns and the 2008/9 campaigns with an all-W wall. A regression $\left(\mathrm{H}_{98(\mathrm{y}, 2)} \propto v_{e}^{*-0.19}\right)$ to all early heated IPHMs without $\mathrm{N}$ seeding is shown as well. Almost all $\mathrm{N}$ seeded data points are above this curve demonstrating the improved confinement found by $\mathrm{N}_{2}$ puffing, introduced for divertor protection. Low collisionality values of previous campaigns are so far not accessible in the all-W AUG. Keeping in mind the high values of $\mathrm{n}_{\mathrm{e}}, \mathrm{f}_{\mathrm{GW}}, v_{e}^{*}$ necessary to achieve stable operation with the all-W AUG, the performance of the 2008/9 N seeded IPHMs is at least as good as in the carbon dominated era, for this parameter range. Similar comparisons of confinement data from several AUG campaigns for IPHMs with higher triangularity $\delta$ or 
lower safety factor $\mathrm{q}_{95}$ show similar trends [9] as presented in fig. 1.

\section{Detailed study of nitrogen seeded Improved H-Modes}

In order to study the effect of $\mathrm{N}$ seeding on energy confinement, discharges with zero nitrogen puffing are compared to $\mathrm{N}$ seeded discharges changing no other parameter. Time traces for such a pair of comparable discharges are given in fig. 2. The chosen pair of discharges (\#24681 and \#24682) was performed during a period in which no boronization for wall conditioning had been applied, but the behaviour presented in fig. 2 is also typical for a boronized AUG. The major difference between boronized and non-boronized conditions is the resulting $\mathrm{N}_{2}$ puff rate used under feedback in real-time to maintain a preset value of the divertor temperature. The $\mathrm{N}_{2}$ puff rate controlled by this feedback system is lower in the nonboronized case, in which the edge and divertor radiation by intrinsic impurities is higher, as compared to conditions with freshly boronized walls.

With the introduction of $\mathrm{N}$ a clear increase in stored energy $\mathrm{W}$ and in $\mathrm{H}_{98}$ is observed which is strongest in the phase with the highest applied heating power $(t \geq 4 \mathrm{~s}$ in fig. 2). This is typical for IPHM discharges where the $\mathrm{H}_{98}$ factor increases with $\beta_{\mathrm{N}}$ [10]. Similar neutron rates (not shown) are found in comparable discharges with and without $\mathrm{N}_{2}$ puff. At given heating power, the higher plasma temperature in the presence of $\mathrm{N}$ compensates the loss in neutron yield due to increased $\mathrm{D}$ dilution in the case with $\mathrm{N}$ seeding [11]. Within the resolution of the MSE diagnostic, q-profiles for comparable discharges with and without $\mathrm{N}$ are identical.

The characteristics of ELMs change with the introduction of nitrogen [12]. The ELM frequency increases, typically by a factor of two, while the relative pedestal energy loss per ELM is on average just half compared to the unseeded case. However, the observed changes in the ELM behaviour cannot explain the better confinement in the presence of nitrogen.

Very similar line-averaged densities (see fig. 2 ) in both discharges suggest that the rise in stored energy in the presence of $\mathrm{N}$ is mainly due to higher temperatures. Radial profiles of electron temperature and density are plotted in fig. 3 for the pair of discharges $\# 24681 / \# 24682$. These profiles are averaged in a phase of highest heating power $(4.2 \mathrm{~s} \leq \mathrm{t} \leq 4.5 \mathrm{~s})$ omitting all data points during ELMs. All available diagnostics (Thomson scattering, ECE, lithium beam, reflectometry and interferometry) were used to determine spline fits of electron temperature and density profiles. The applied fit procedure was exactly the same for the two consecutive discharges. Therefore, the relative changes between the unseeded and the seeded case should be reflected by the presented profiles with the highest possible accuracy. The radial range has been restricted to the plasma edge region which is 
thought to be decisive for the characterisation (analysis) of the observed confinement improvement. While the electron density profiles are to first order independent on the presence of nitrogen, the electron temperature is significantly higher at the pedestal top. The steep gradient zone seems to extend further inside in the case with $\mathrm{N}$ seeding which leads to higher temperature at $\rho=0.85$. Further inside $(\rho \leq 0.85)$ the temperature gradient lengths of the unseeded and seeded discharge are identical. Gyrokinetic calculations [13] identify the Ion Temperature Gradient (ITG) driven mode as the dominant instability in the plasma core. Under these circumstances, profile stiffness is expected, so that the inverse gradient length $R / L_{T}=R \cdot|\nabla T| / T$ (of electrons and ions) is close to its critical value [13].

Non-linear gyro-kinetic simulations of the core transport using GENE showed in the $\mathrm{N}$ seeded case a shift of the ITG critical $\mathrm{R} / \mathrm{L}_{\mathrm{Ti}}$ towards higher values. This is caused by the reduction of the ITG mode due to deuterium dilution [9]. However, this effect, which would otherwise point to a core confinement improvement in the presence of $\mathrm{N}$, is completely compensated by the observed higher $\mathrm{T}_{\mathrm{e}}$, values of $\mathrm{N}$ seeded discharges, since the heat flux grows above the critical $\mathrm{R} / \mathrm{L}_{\mathrm{Ti}}$ as $\mathrm{T}_{\mathrm{i}}^{5 / 2}$. (More details of the linear and non-linear simulations of core transport will be published elsewhere [14]). Therefore, the overall confinement improvement is mainly due to a broader H-mode pedestal leading to higher pedestal top temperatures which extend to the core via profile stiffness.

In fig. $4, Z_{\text {eff }}$ profiles are plotted for the pair of discharges \#24681/\#24682. The concept of Integrated Data Analysis (IDA) within the framework of Bayesian probability theory was applied to determine the $Z_{\text {eff }}$ profiles by combined analysis of background emission measured by the CXRS diagnostic and bremsstrahlung data [15]. The observed changes of the radial $Z_{\text {eff }}$ profile by the introduction of $\mathrm{N}$ suggest a flat to slightly hollow nitrogen density profile, which still needs to be confirmed by CXRS nitrogen density profile measurements. With the introduction of nitrogen the change in the measured $Z_{\text {eff }}$ profile leads to a pronounced increase of $\mathrm{D}$ dilution by around $25 \%$ at the edge transport barrier $\left(\rho_{\mathrm{pol}}=0.9\right)$, while in the plasma core, the $\mathrm{D}$ dilution increases only by 5\% [9]. Assuming an average charge of 7 for the dominant impurity ion in the unseeded (average of fully stripped oxygen and carbon) and the seeded case (fully stripped nitrogen), one can calculate deuterium density profiles (see fig. 4) using the fitted electron density profiles of fig. 3 (full) and the presented $Z_{\text {eff }}$ profiles. In fig. 4 , the increased $\mathrm{D}$ dilution at the plasma edge caused by nitrogen ions implies higher ion temperatures to keep the same pedestal D ion pressure as in the unseeded case (see fig. 5). Ion pressure profiles in fig. 5 are calculated with deuterium density profiles of fig. 4 and the 
assumption $T_{i}=T_{e}$. ( $T_{e}$ profile data from fig. 3). In combination with a diminishing rise of dilution in the core [9] caused by the $\mathrm{N}_{2}$ puff and the well-established stiffness of $\mathrm{T}_{\mathrm{i}}$ profiles, a higher core $\mathrm{D}$ ion pressure of $\mathrm{N}$ seeded IPHMs (i.e. improved confinement) can be understood.

At the high pedestal densities the energy exchange between ion and electrons is rather effective - the equipartition time between $T_{e}$ and $T_{i}$ is about one order of magnitude shorter than the thermal energy confinement time $\tau_{t h}$ - and similar values of $T_{i}$ and $T_{e}$ are measured [12]. Therefore, electron temperature is also affected by the introduction of $\mathrm{N}$ and higher electron pressure (see fig. 5) is measured for $\rho \leq 0.93$ in the seeded case. In the steep gradient region $(\rho>0.93)$, however, the electron pressure profiles of both discharges are the same. For completeness also the electron collisionality (same definition of $v_{e}^{*}$ as in [11]) is plotted in fig. 5. No significant changes are visible. In the $\mathrm{N}$ seeded discharge, the higher $\mathrm{Z}_{\text {eff }}$ is compensated by higher $\mathrm{T}_{\mathrm{e}}$ values.

In fig. 6 , electron temperature and density values close to the pedestal top $\left(\rho_{\mathrm{pol}}=0.9\right)$ are plotted for a set of discharges similar to the presented pair \#24681/\#24682 in order to document the robustness of the found effects. While the range of pedestal densities is not affected at all by the introduction of $\mathrm{N}$, the pedestal electron temperature $\mathrm{T}_{\mathrm{e}}$ of $\mathrm{N}$ seeded discharges is considerably higher than in the unseeded case. A similar effect has been found for $T_{i}$ (not shown) for a smaller data set.

A strong correlation between $\mathrm{Z}_{\text {eff }}$ values and the $\mathrm{H}_{98}$ factor for IPHMs with and without $\mathrm{N}$ seeding was found in [15]. Here an attempt is made to highlight the important role of the $Z_{\text {eff }}$ parameter for the scaling of the thermal energy confinement time $\tau_{\text {th }}$ (see fig. 7). Together with heating power $P_{\text {heat }}$ and the average $Z_{\text {eff }}$ a power law fit $\tau_{t h} \propto P_{\text {heat }}^{-0.5} \cdot Z_{\text {eff }}^{0.5}$ to a subset of 2008/9 IMPH data was established which describes the experimental $\tau_{\text {th }}$ data rather well. Inclusion of additional parameters did not improve the fit. In particular, no dependence of $\tau_{\text {th }}$ on electron density $n_{e}$ was found. This is in agreement with the much larger dataset of fig. 1 where normalized confinement $\mathrm{H}_{98(\mathrm{y}, 2)}$ factors are plotted on the ordinate instead of confinement times $\tau_{\text {th }}$ as in fig. 7. As stated in section 2, the obvious degradation of normalized confinement $\mathrm{H}_{98(\mathrm{y}, 2)}$ with increasing density in fig. 1 reflects just the positive density dependence of the H-mode scaling $\left(\tau_{98(\mathrm{y}, 2)} \propto \mathrm{n}_{\mathrm{e}}{ }^{0.41}\right)$, while $\tau_{\text {th }}$ values do not show such a dependence.

The weaker degradation of $\tau_{\text {th }}$ with $\mathrm{P}_{\text {heat }}\left(\propto \mathrm{P}_{\text {heat }}{ }^{-0.5}\right)$ compared to the H-mode scaling $\tau_{98(\mathrm{y}, 2)}(\propto$ $\mathrm{P}_{\text {heat }}{ }^{-0.69}$ ) reflects the observed increase of $\mathrm{H}_{98(\mathrm{y}, 2)}$ with $\beta_{\mathrm{N}}$ typical for the IPHM scenario [10]. 


\section{Summary and Conclusions}

In the 2008/9 campaigns high performance improved H-mode discharges were conducted with good energy confinement in the all-tungsten AUG. The energy confinement was most improved when nitrogen was puffed with the primary goal of cooling the plasma edge. The achieved confinement factors $\mathrm{H}_{98}$ turned out to be as good as, or even better than, the values obtained in the carbon dominated AUG when discharges at the same values of $\mathrm{f}_{\mathrm{GW}}$ are compared. Thus, the compatibility of ITER relevant high confinement modes such as the improved H-mode, or Hybrid scenario, with an all-W wall has been well demonstrated in ASDEX Upgrade. Better IPHM energy confinement was found in previous AUG campaigns $(<2006)$ but only at lower $v^{*}$ which has, so far, not been accessible in the all-W AUG. It is hoped that both short- and long-term improvements (solid tungsten outer divertor with increased pumping capability, more ECRH power) will expand the IPHM operational space to lower collisionalities.

The improved confinement of $\mathrm{N}$ seeded IPHMs appears to be an effect of higher pedestal temperatures which extend to the plasma core via profile stiffness. $Z_{\text {eff }}$ (determined with integrated data analysis methods) turned out to be an important parameter to quantify the influence of nitrogen seeding on the energy confinement in the all-W AUG. The pronounced dilution at the plasma edge by nitrogen allows higher ion temperatures at the same edge ion pressure as in the unseeded case, while the dilution of the core plasma remains moderate. The dilution effect in the edge transport barrier up to the pedestal top seems to play an important role which unfortunately cannot be modelled with present day code packages. Better theoretical understanding of the H-mode transport barrier in general will be required to fully understand the observed effect of confinement improvement which clearly has its origin in the plasma edge as was shown in this paper.

Peaking of the density profile in the presence of $\mathrm{N}$ is weak and gives only a small contribution to the confinement improvement. This is a major difference to previously discovered regimes with improved confinement following impurity seeding using predominately $\mathrm{Ne}$ and $\mathrm{Ar}$ (TEXTOR: RI-Mode [16] and AUG: CDH-Mode [17]) where density peaking was a dominant contributor to the confinement improvement.

In recent years, nitrogen was also used on ALCATOR C-Mod [18] and JET [19] to moderate divertor heat loads. Experiments on ALCATOR C-Mod with its metal wall were conducted in the (Enhanced D-alpha) EDA H-mode regime while on JET type-I ELMy H-modes in a high triangularity shape were executed. The ALCATOR C-Mod results demonstrate good energy confinement $\left(\mathrm{H}_{98} \approx 1\right)$ in $\mathrm{N}_{2}$ seeded discharges with a highly radiating divertor and "standard" 
core plasma density profiles without enhanced peaking [20]. In the JET device strongly reduced target heat loads could be achieved with $\mathrm{N}_{2}$ puffs while the loss in normalized energy confinement was kept below 10\% [19]. These JET experiments were done at more than 90\% of the Greenwald density. Working so close to the density limit might explain the somewhat low confinement in terms of $\mathrm{H}$ factors $\left(0.9<\mathrm{H}_{98}<1\right)$.

Results on ALCATOR C-Mod, ASDEX Upgrade and JET have proven that nitrogen is well suited and even better than other radiators like Ne or Ar to reach simultaneously moderate divertor heat flux and good confinement. This suggests that for keeping high confinement in radiative scenarios the majority of radiation induced by the external impurity should be produced in the scrape-off layer and divertor rather than on closed flux surfaces.

While on all three machines ALCATOR C-Mod, ASDEX Upgrade and JET well-confined plasmas with nitrogen seeding could be produced, a robust and significant effect of confinement improvement (up to 25\%) has only been observed at ASDEX Upgrade until now. The reason for this is unclear. Further studies will be required, in particular experiments during the 2011/12 JET campaigns with the ITER-like Wall [21] which will make results from AUG and JET more comparable and will hopefully lead to new insights.

\section{References}

[1] STOBER, J., et al., Nucl. Fusion 47 (2007) 728-737

[2] NEU, R., for the ASDEX Upgrade team, Plasma Phys. Contr. Fus. 49 (2007) B59

[3] DUX, R., et al., Nucl. Fusion 51 (2011), 053002

[4] GRUBER, O., et al., Nucl. Fusion 49 (2009) 115014

[5] BOBKOV, V., et al. Nucl. Fusion 50 (2010) 035004

[6] NEU, R., et al., 'Power and Particle Exhaust Control in All W ASDEX Upgrade', in Fusion Energy 2010 (Proc. 23rd Int. Conf. Daejeon, 2010) (Vienna: IAEA) CD-ROM file EXD/P3-24 and http://www-pub.iaea.org/MTCD/Meetings/PDFplus/2010/cn180/cn180_papers/exd_p3-24.pdf

[7] KALlENBACH, A., et al., Plasma Phys. Contr. Fus. 52 (2010) 055002

[8] CHRISTIANSEN, J. P., et al., Nucl. Fusion 30 (1990) 1183

[9] MAGGI, C. F., et al. Nucl. Fusion 50 (2010) 025023

[10] TARDINI, G., et al., 'Confinement enhancement in ASDEX Upgrade improved Hmode discharges with nitrogen seeding', presented at the $36^{\text {th }}$ EPS Conference on Plasma Phys. Sofia, June 29-July 3, 2009, http://epsppd.epfl.ch/Sofia/pdf/O2_004.pdf

[11] WOLfRUM, E., et al., Plasma Phys. Control. Fusion 53 (2011) 085026

[12] TARDINI, G., et al., 'Core transport and pedestal characteristics of nitrogen seeded Hmode discharges in ASDEX Upgrade', presented at the $37^{\text {th }}$ EPS Conference on Plasma Phys. Dublin, June 21-25, 2010, P1.1097, http://ocs.ciemat.es/EPS2010PAP/pdf/P1.1097.pdf

[13] SCHWEINZER, J., et al., 'Confinement of 'Improved H-Modes' in the All-Tungsten ASDEX Upgrade’, Fusion Energy 2010 (Proc. 23rd Int. Conf. Daejeon, 2010) (Vienna: 
IAEA) CD-ROM file EXC/P2-07 and http://www-pub.iaea.org/MTCD/Meetings/PDFplus/2010/cn180/cn180_papers/exc_p2-07.pdf

[14] TARDINI, G., et al., Core transport analysis of nitrogen seeded H-mode discharges in ASDEX Upgrade, 2011, submitted to Plasma Phys. Contr. Fus.

[15] RATHGEBER, S. K., et al., Plasma Phys. Contr. Fus. 52 (2010) 095008

[16] MESSIAEN, A. M., et al., Nucl. Fusion, 34 (1994) 825

[17] GRUBER, O., et al., Phys. Rev. Lett. 74 (1995) 4217

[18] REINKE, M.L., et al. J. Nucl. Mater. (2010), doi:10.1016/j.jnucmat.2010.10.055

[19] MADDISON, G.P., et al. Nucl. Fusion 51 (2010) 042001

[20] LOARTE, A., et al. Physics of Plasmas 18, (2011) 056105

[21] MATTHEWS, G., et al. Phys. Scr. T138 (2009) 014030

Figures \& Captions:
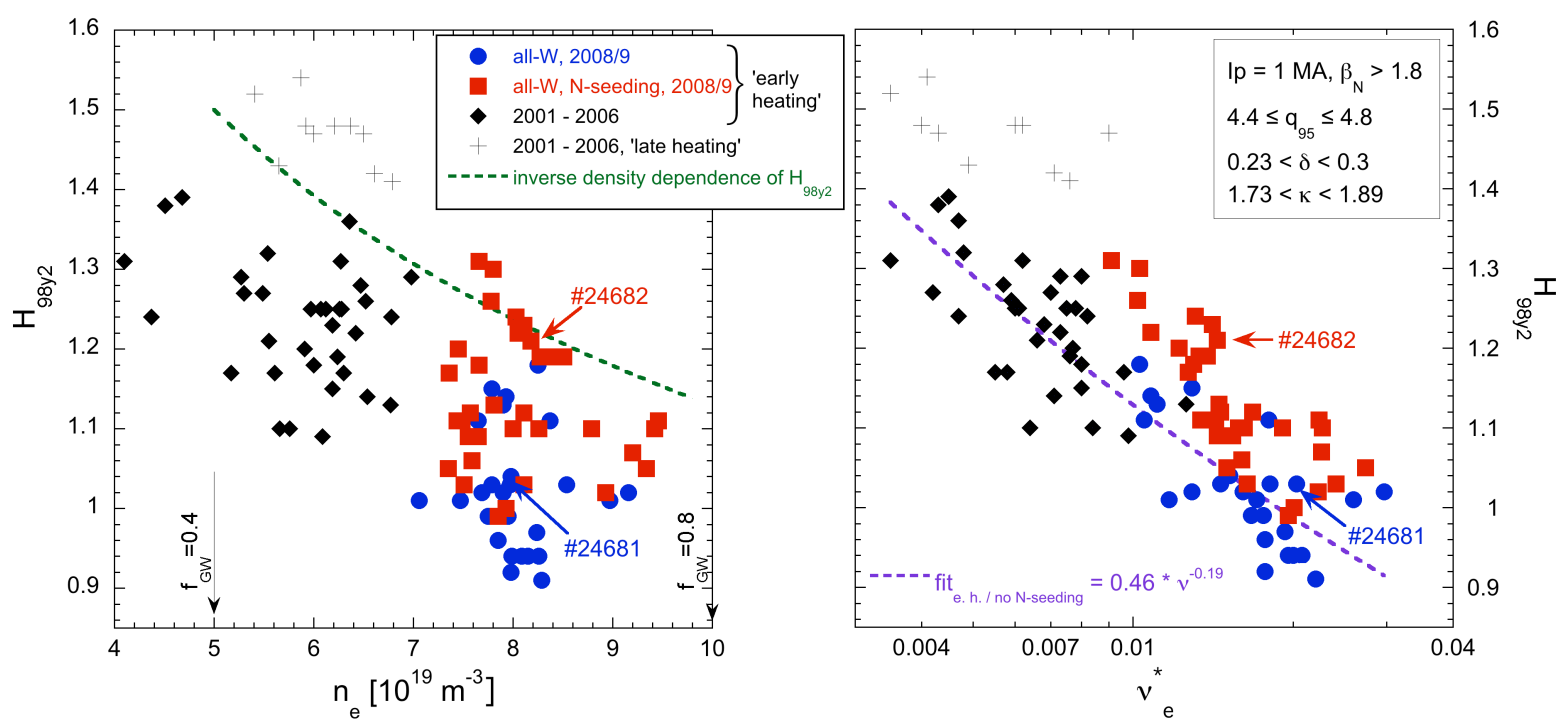

FIG. 1. H-mode confinement enhancement factor $H_{98(y, 2)}$ dependence on line-averaged density (left) and collisionality (right) for 'early' (full symbols) and 'late' (crosses) heated improved H-mode discharges with plasma currents of $1 M A$ at small/medium triangularity $(0.23<\delta<0.3)$ with a $q_{95} \sim 4.6$. The presented data covers a range in Greenwald fraction $f_{G W}$ from 0.35 to 0.75 (see left figure).

The data have a total additional heating power between 5-15MW. Discharges from the 2008/9 campaigns (all-W AUG) are with $D_{2}$ fuelling $\left(1.0 \cdot 10^{21}-1.2 \cdot 10^{22} \mathrm{~s}^{-1}\right)$ while discharges from 2001 to 2006 have no gas fuelling except a single one $\left(7 \cdot 10^{21} \mathrm{~s}^{-1}, H_{98 y 2}=1.1, n_{e}=7 \cdot 10^{19} \mathrm{~m}^{-3}\right)$. In 2008/9 all discharges use the early heating approach and have at least 0.8MW of ECRH power. The blue full circles refer to operation without $N$ seeding, while the red points are with $N$ seeding. The data points of discharges) used for the detailed comparison of non-seeded (\#24681) with N seeded (\#24682) discharges are indicated. 

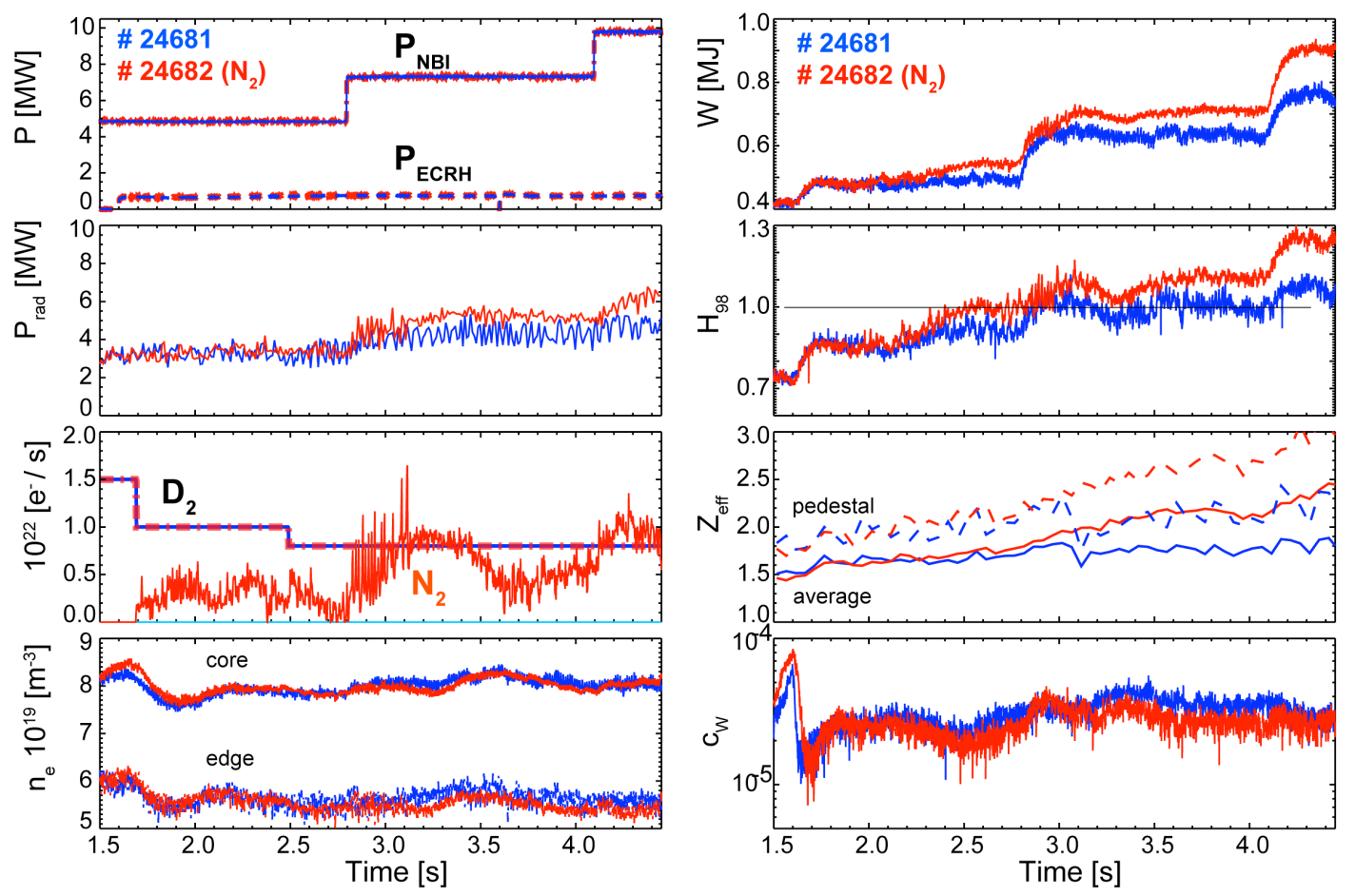

FIG. 2. Time traces of various parameters for two consecutive discharges (non-boronized AUG, $\left.I_{p}=1 M A, B_{t}=2.5 T, q_{95}=4.6, \delta=0.29\right)$ with $(\# 24682$, red $)$ and without $\left(\# 24681\right.$, blue) $N$ seeding. The $N_{2}$ puff rate is feedback controlled to keep a preset divertor temperature (not shown). All discharge parameters are kept the same for both discharges, except to the introduction of $N$ in the case of \#24682. Stored energy $W, H$ factor $H_{98}$, total radiation $P_{\text {rad }}$ and $Z_{\text {eff (line averaged and pedestal value) }}$ increase with the applied $N_{2}$ puff, while other parameters like neutron yield (not shown), lineaveraged density $n_{e}$ and tungsten concentration $c_{W}$ remain the same. 


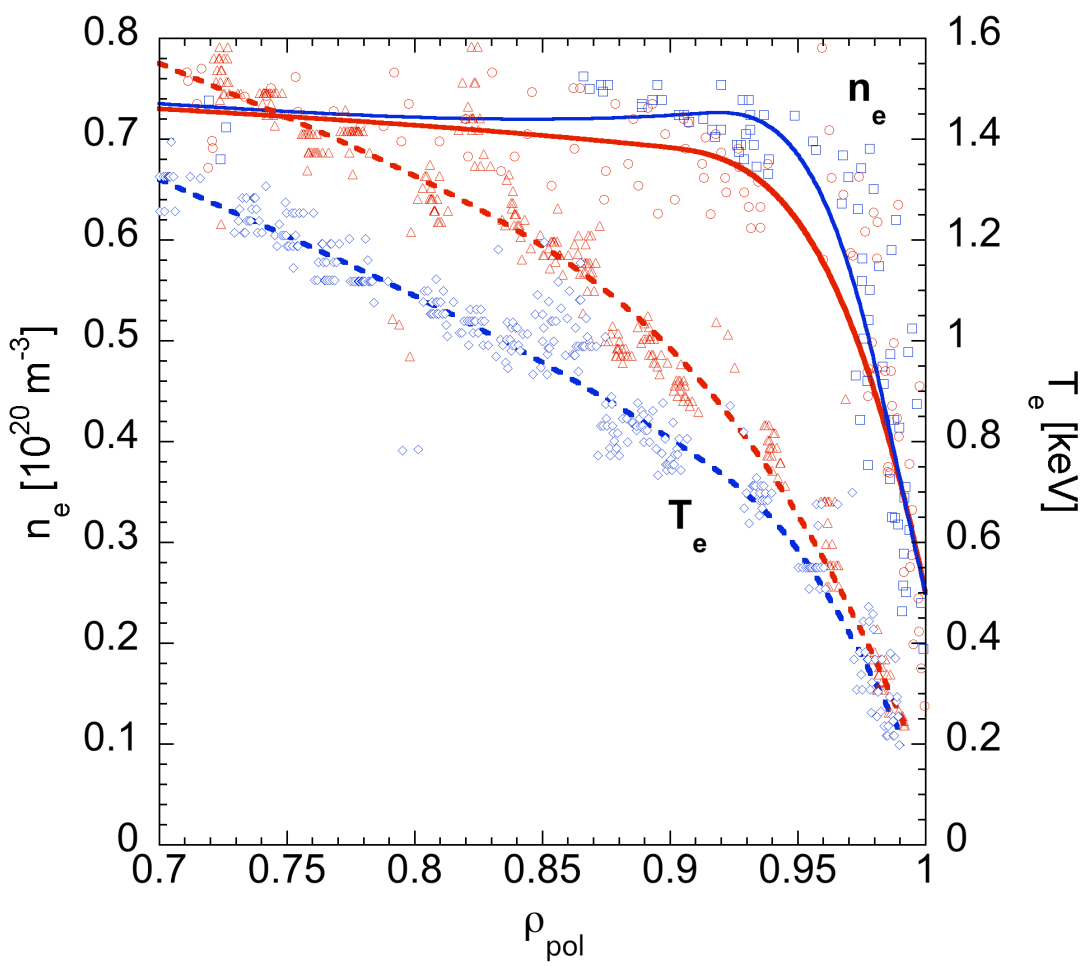

FIG. 3: Electron temperature $T_{e}$ (right axis) and density profiles $n_{e}$ (left axis) of the AUG plasma edge for unseeded (blue, \#24681) and nitrogen seeded (red, \#24682) improved H-modes (see also fig. 2). Symbols represent data points of ECE, Thomson scattering, reflectometry and lithium beam diagnostics. Only data in-between ELMs has been taken in the time interval $4.2 \mathrm{~s} \leq t \leq 4.5 \mathrm{~s}$. In addition, spline fits ( $T_{e}$ : dotted lines, $n_{e}$ : solid lines) to the data points are shown. For the electron density fit also the edge channel of the DCN interferometry diagnostic has been taken into account. 


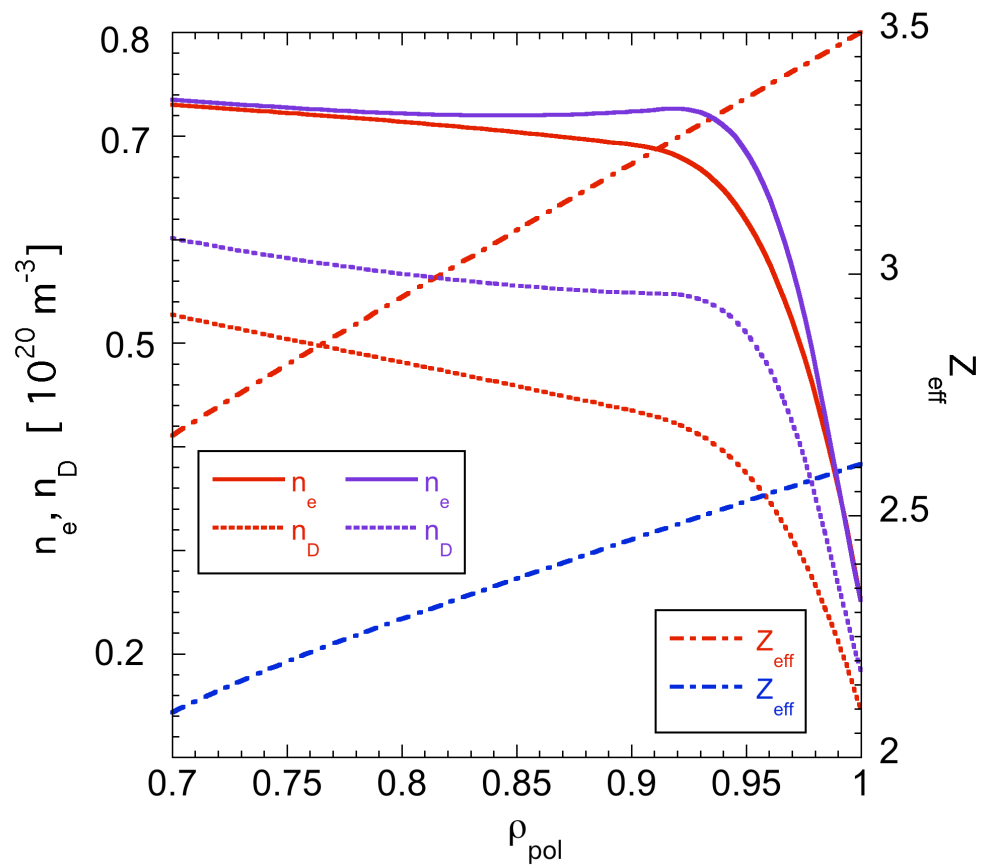

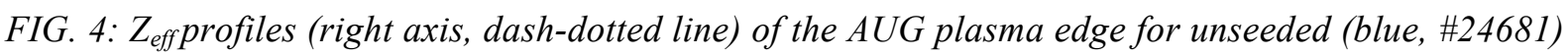
and nitrogen seeded (red, \#24682) improved H-modes (see also fig. 2). These profiles originate from integrated data analysis (IDA). Assuming an average charge of 7 for the dominant impurity ion in the unseeded (average of fully stripped oxygen and carbon) and the seeded case (fully stripped nitrogen), one can calculate deuterium density profiles (dotted line) using the fitted electron density profiles of fig. 3 (solid line) and the presented $Z_{\text {eff }}$ profiles.

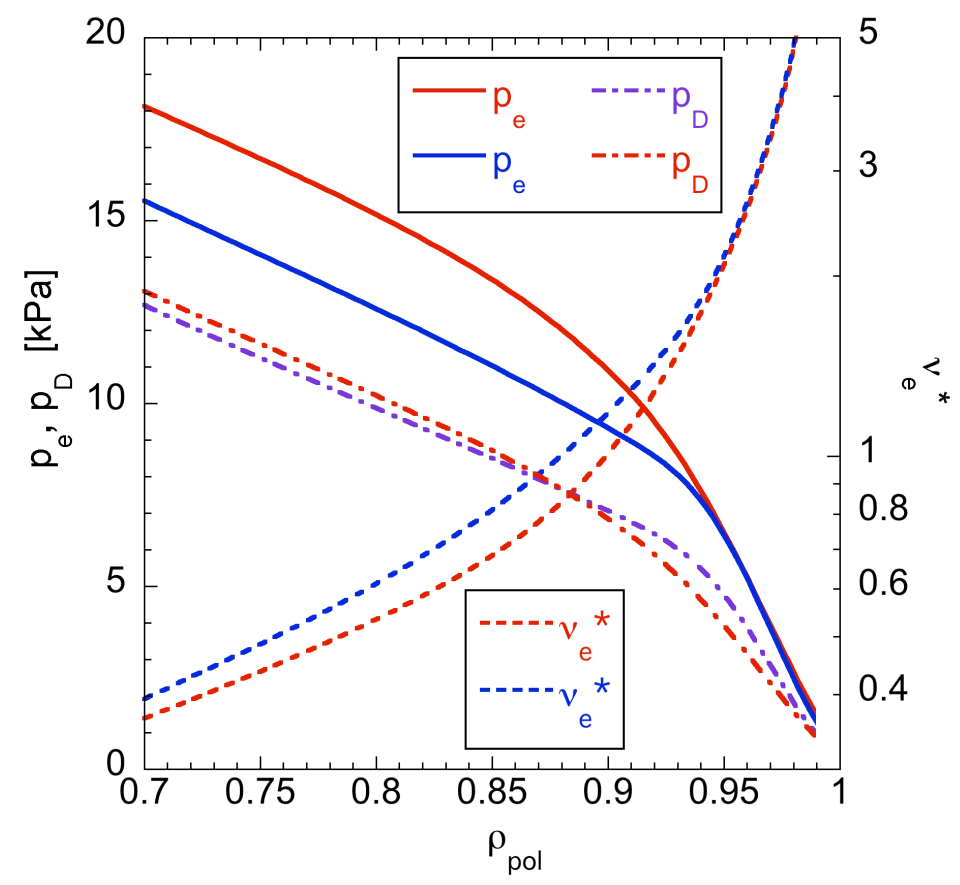

FIG. 5: Profile data of figs. 5 and 6 combined to calculate electron pressure (solid) and electron collisionality (dotted) profiles for unseeded (blue, \#24681) and nitrogen seeded (red, \#24682) 
improved H-modes. In addition, the D ion pressure (dash-dotted) is calculated with $n_{D}$ data from fig. 4 and the assumption $T_{i}=T_{e}$ ( $T_{e}$ profile data from fig. 3 ).

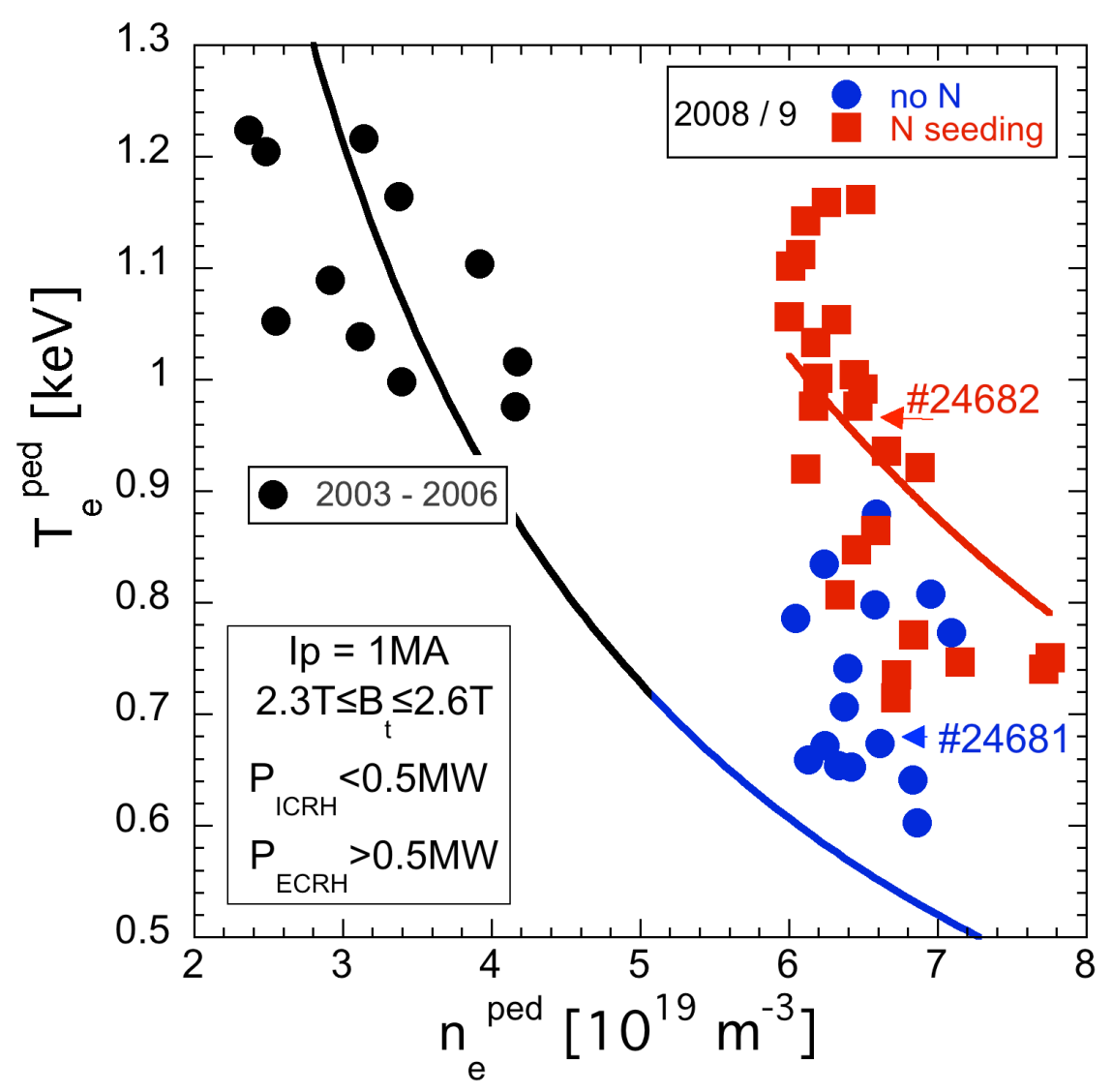

FIG. 6. Electron temperature and density values close to the pedestal top $\left(\rho_{p o l}=0.9\right)$. All results of the 2008/9 all-W campaigns are at densities above $5.5 \cdot 10^{19} \mathrm{~m}^{-3}$ and well separated from the lower ones of previous campaigns (black symbols). Puffing of $\mathrm{N}_{2}$ (red symbols) does not change the typical pedestal density of IPHMs, but increases the temperature. This indicates an increase of the electron pressure at the pedestal top. Hyperbolic fits of $T_{e}$ to both the unseeded (black-blue) and seeded (red) dataset indicate lines of constant pressure. 


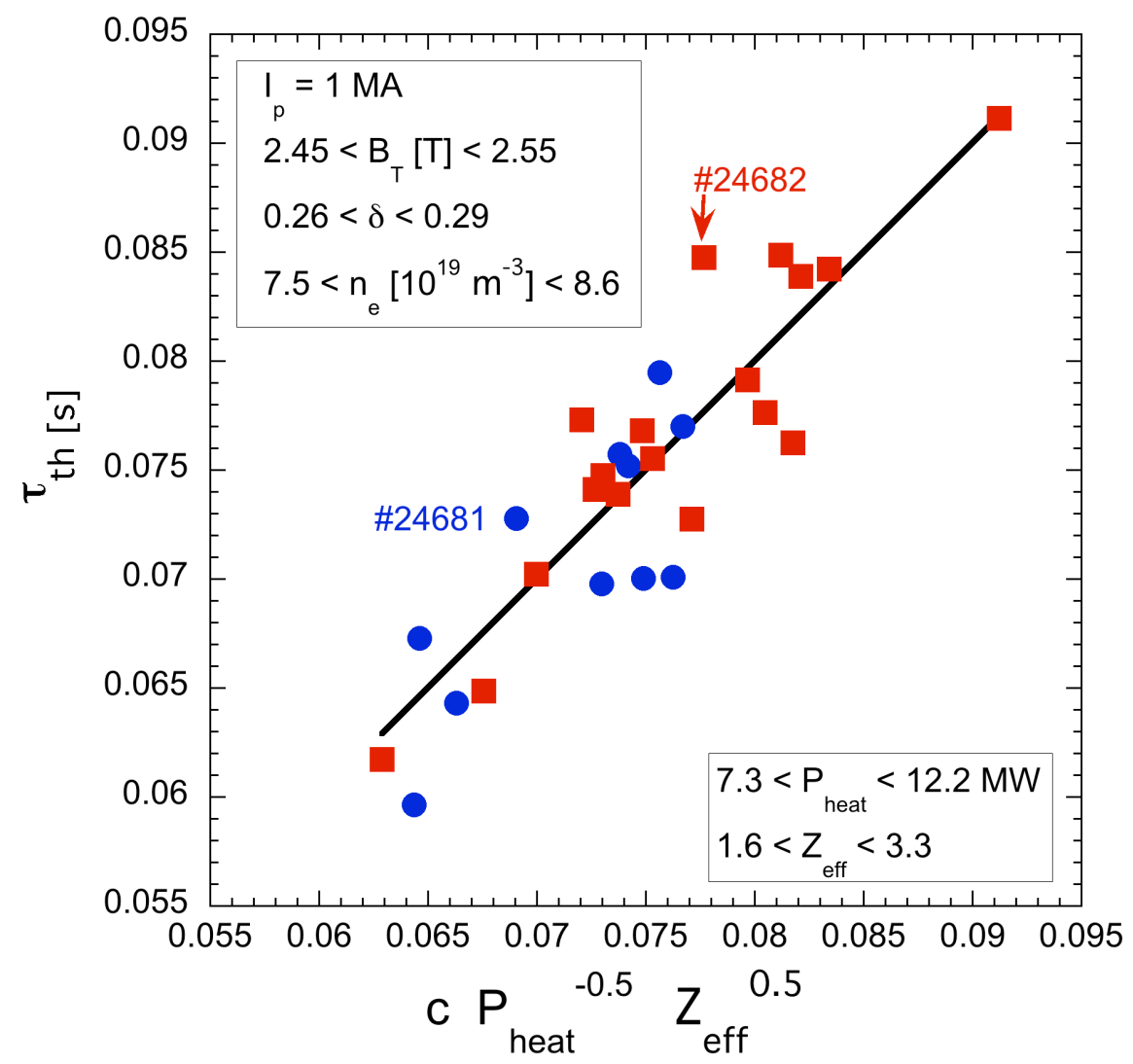

FIG. 7. Fit of thermal energy confinement $\tau_{\text {th }}$ for IPHMs of $I_{p}=1 M A$ and $B_{t}=2.5 T$ (dataset from 2008/9 where $Z_{\text {eff }}$ values from IDA diagnostic were available). A power law Ansatz with heating power $P_{\text {heat }}$ and line-averaged $Z_{\text {eff }}$ leads to a good description of the data. The power degradation of confinement is less than in $H_{98(y, 2)}$. 